\title{
Reaction of $\delta$-Tocopherol with an Alkylperoxyl Radical
}

\author{
Ryo Yamauchi, Tomoatsu Matsui, Nobuyuki Mryake, \\ Koji Kato and Yoshimitsu UENo* \\ Department of Food Science, Faculty of Agriculture, \\ Gifu University, Gifu 501-11, Japan
}

Received June 20, 1990

\begin{abstract}
$\delta$-Tocopherol was reacted with an alkylperoxyl radical at $37^{\circ} \mathrm{C}$ in benzene, $2,2^{\prime}$-Azobis $(2,4-$ dimethylvaleronitrile) was used to generate the alkylperoxyl radical. The reaction products of $\delta$-tocopherol were isolated and characterized. There were $\delta$-tocored, four stereoisomers of 8a(1-cyano-1,3-dimethyl)butylperoxy- $\delta$-tocopherone, a mixture of four stereoisomers of 8a-(1-cyano1,3-dimethyl)butylperoxy-5-( $\delta$-tocopheroxy)- $\delta$-tocopherone, 5-( $\delta$-tocopheroxy)- $\delta$-tocopherol, a mixture of four stereoisomers of 8a-(1-cyano-1,3-dimethyl)butylperoxy-5-[(5-( $\delta$-tocopheroxy)- $\delta$-tocopheroxy $]-\delta$-tocopherone, and 5 -[(5-( $\delta$-tocopheroxy)- $\delta$-tocopheroxy $]-\delta$-tocopherol. From the reaction products of $\delta$-tocopherol and its dimer, a possible mechanism for the reaction of $\delta$-tocopherol with peroxyl radicals was discussed.
\end{abstract}

Tocopherols are natural antioxidants in foods and are important in promoting the stability of vegetable oils. Tocopherols inhibit autoxidation of oils by trapping peroxyl radicals. $^{1,2)}$ To elucidate the antioxidative mechanisms of tocopherols, the reaction products of tocopherols with peroxyl radicals have been extensively studied. ${ }^{3-9)}$ We have already reported that the reactions of $\alpha$ - and $\gamma$-tocopherols with an alkylperoxyl radical generated from the thermal decomposition of a radical initiator, 2,2'-azobis (2,4-dimethylvaleronitrile) (AMVN), give the 8a-alkylperoxy tocopherones and some other compounds. ${ }^{5,7,9)}$ $\delta$-Tocopherol is also present in vegetable oils. ${ }^{10,11)}$ Although the oxidation products of $\delta$-tocopherol during the autoxidation of unsaturated lipids have been reported to be some reducing dimers, ${ }^{12,13)}$ the reaction of $\delta$-tocopherol with lipid-peroxyl radicals has not been satisfactorily elucidated. This paper reports the reaction products of $\delta$-tocopherol with AMVN in benzene.

\section{Materials and Methods}

Materials. RRR- $\delta$-Tocopherol was prepared from mixed isomers of tocopherol (Hohnen Oil Co.). ${ }^{14)} \mathrm{A}$ free radical initiator, AMVN, was purchased from Wako Pure Chem. Ind. $\delta$-Tocopherylquinone was prepared from $\delta$-tocopherol by oxidation with $\mathrm{FeCl}_{3}{ }^{15}{ }^{5} \mathrm{~A} \delta$-tocopherol dimer, 5 -( $\delta$-tocopheroxy $)$ - $\delta$-tocopherol $(\delta$-tocopherol diphenylether dimer: $\delta$-TED), was isolated from the reaction products of $\delta$-tocopherol with $p$-benzoquinone. ${ }^{16)} 2,2,5,7,8$ Pentamethylchroman-6-ol (PMC) was prepared by the method of Nilsson et al. ${ }^{17)}$ All other chemicals were of analytical grade.

High performance liquid chromatography ( $H P L C$ ). HPLC was done with a Jasco Trirotar V pump equipped with a Model GP-A40 gradient programmer. A Jasco Model 875-UV detector or a Model 820-FP spectrofinorometer was used as the detector.

Reaction procedure. $\delta$-Tocopherol $(0.83 \mathrm{mg}, 2 \mathrm{mmol})$ and AMVN $(49.6 \mathrm{~g}, 200 \mathrm{mmol})$ were dissolved in benzene $(1000 \mathrm{ml})$ and incubated at $37^{\circ} \mathrm{C}$ for 45 or $90 \mathrm{~min}$ under air. After the solvent was removed in vacuo, the oily residue was dissolved in $200 \mathrm{ml}$ of ethanol and left at $-20^{\circ} \mathrm{C}$ for $2 \mathrm{hr}$. The resulting crystals of AMVN were removed by filtration. The reaction products in the filtrate were isolated by preparative reverse-phase HPLC. A Wakosil 5C18 column $(10 \times 300 \mathrm{~mm}$, Wako Pure Chem. Ind.) was developed with methanol at a flow rate of $5.0 \mathrm{ml} / \mathrm{min}$. Then, the dimer and trimer in the column were eluted with methanol-ethyl acetate $(3: 2, \mathrm{v} / \mathrm{v}$ for dimer and $3: 7, \mathrm{v} / \mathrm{v}$ for trimer, respectively). The products were further purified by normal-phase HPLC. A LiChrosorb Si60 column $(4.6 \times 250 \mathrm{~mm}$, Merck) was developed with hexane-2-

* Present address: Laboratory of Food Chemistry, Tokai Women's Junior College, Kakamigahara, Gifu 504 , Japan. 
propanol $(98: 2, \mathrm{v} / \mathrm{v})$ at a flow rate of $1.5 \mathrm{ml} / \mathrm{min}$. The eluents were monitored by its absorbance at $285 \mathrm{~nm}$. The purities of isolated compounds were checked by thin layer chromatography on pre-coated silica gel 60 plate (Merck) using hexane-diethyl ether $(70: 30, v / v)$. The isolated compounds were dissolved in ethanol or hexane and stored at $-20^{\circ} \mathrm{C}$ until analysis.

To observe the course of the reaction, $\delta$-tocopherol $(0.73 \mathrm{~mm}$ or $22.5 \mathrm{~mm})$ or $\delta$-TED (1.22 mM) was reacted with AMVN (100 mM) in benzene at $37^{\circ} \mathrm{C}$. At regular intervals, a $0.5 \mathrm{ml}$-sample was withdrawn and the amounts of $\delta$-tocopherol and its reaction products were measured by reverse-phase $\mathrm{HPLC}$. A $\mu$ Bondasphere $5 \mu \mathrm{C} 18100 \AA$ column $(3.9 \times 150 \mathrm{~mm}$, Nihon Waters Co. $)$ was developed with a methanol or a 15-min linear gradient of methanol to methanol-ethyl acetate $(3: 7, v / v)$ at a flow rate of $1.0 \mathrm{ml} / \mathrm{min}$. The amounts of $\delta$-tocopherol were calculated from the peak area by monitoring the fluorescence (ex., $290 \mathrm{~nm}$; em. $330 \mathrm{~nm}$ ). PMC was used as an internal standard. The amounts of the reaction products were calculated from the peak area by monitoring the elutions at $240 \mathrm{~nm}$ or $290 \mathrm{~nm}$. $\delta$-Tocopherylquinone was used as internal standards.

Spectroscopy. Proton $\left({ }^{1} \mathrm{H}\right)$ and carbon- $13\left({ }^{13} \mathrm{C}\right)$ nuclear magnetic resonance (NMR) spectra were recorded on a Jeol JNM-GX-270 FT NMR spectrometer. Mass spectra (MS) were obtained with a Shimadzu GCMS 9020 DF gas chromatograph-mass spectometer system with an ionizing energy of $70 \mathrm{eV}$. Infrared (IR) spectra of samples were taken on a Jasco A-302 IR spectrometer. Ultraviolet (UV) spectra were measured with a Jasco Ubest-30 spectrophotometer. Specific rotations were measured with a Union PM-201 polarimeter.

\section{Results}

$\delta$-Tocopherol and AMVN were reacted at $37^{\circ} \mathrm{C}$ for 45 or $90 \mathrm{~min}$, and the reaction products were analyzed by reverse-phase HPLC (Fig. 1). Many peaks corresponding to the reaction products appeared on the chromatograms. Peaks 1, 2, 3, 4, 5 and 7 in the 90 -min reaction mixture and peaks 6 and 8 in the 40 -min reaction mixture could be isolated by preparative reverse-phase HPLC developed with increasing proportions of ethyl acetate in methanol. Peak 3 was further separated into two peaks, $\mathbf{3 a}$ and $\mathbf{3 b}$, by normal-phase HPLC. The structures of $\mathbf{1}, \mathbf{2}, \mathbf{3 a}, \mathbf{3 b}, \mathbf{4}, \mathbf{5}, \mathbf{6}, 7$ and $\mathbf{8}$ were identified as described below (Fig. 2).

Compound 1 was obtained as a dark red oil ( $R f 0.21,41.1 \mathrm{mg}$ yield). The structure was

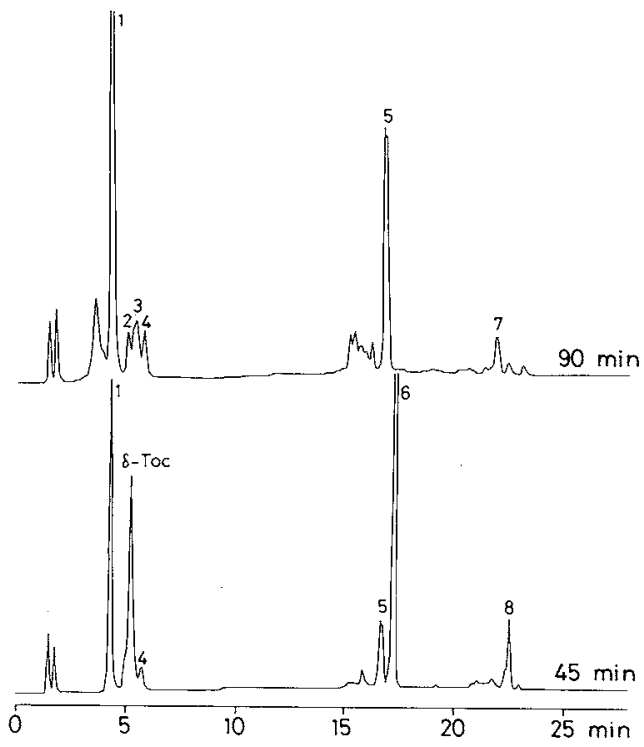

Fig. 1. Reverse-phase HPLC of the Mixture Resulting from the Reaction of $\delta$-Tocopherol with AMVN in Benzene.

$\delta$-Tocopherol (2 mM) and AMVN (200 mM) in benzene solution were incubated at $37^{\circ} \mathrm{C}$ for $45 \mathrm{~min}$ or $90 \mathrm{~min}$. HPLC was done with a $\mu \mathrm{B}$ ondasphere $5 \mu \mathrm{Cl} 8100 \AA$ column developed with a 15 -min linear gradient of methanol to methanol ethyl acetate $(3: 7, \mathrm{v} / \mathrm{v})$ at a flow rate of $1.0 \mathrm{ml} / \mathrm{min}$. The eluent was monitored by its absorbance at $285 \mathrm{~nm}$.

identified to be $\delta$-tocored by comparing its spectral data with tocored. ${ }^{9)} \mathrm{UV} \lambda_{\max }$ (ethanol) $\mathrm{nm}(\varepsilon)$ : 276.5 (5900); IR $v_{\max }$ (film) $\mathrm{cm}^{-1}$ : 1690, 1640, 1590; MS m/z: $416\left(\mathrm{M}^{+}, 69 \%\right), 402$ (3), 221 (13), 173 (16), $182(24), 152(100) ;{ }^{1} \mathrm{H}$ NMR $\delta \quad\left(\mathrm{CDCl}_{3}\right): \quad 0.84-0.88 \quad(12 \mathrm{H}, \mathrm{m})$, $1.11-1.62(21 \mathrm{H}$, broad $\mathrm{m}), 1.33(3 \mathrm{H}, \mathrm{s}), 1.73$ $(2 \mathrm{H}, \mathrm{m}), 2.08(3 \mathrm{H}, \mathrm{s}), 2.43(2 \mathrm{H}, \mathrm{m}), 6.16(1 \mathrm{H}$, s); ${ }^{13} \mathrm{C} \mathrm{NMR} \delta\left(\mathrm{CDCl}_{3}\right): 15.6,17.8,19.6,19.7$, $20.9,22.6,22.7,23.8,24.4,24.8,28.0,29.0$, $32.6,32.8,37.3(2 \mathrm{C}), 37.4(2 \mathrm{C}), 39.4,39.9,81.4$, $112.2,126.6,150.3,162.2,177.9,180.7$.

Compounds 2, 3a, $\mathbf{3 b}$ and $\mathbf{4}$ were obtained as colorless oils (2: $R f$ 0.36, $10.0 \mathrm{mg}$ yield; $\mathbf{3 a}$ : Rf $0.45,10.6 \mathrm{mg}$ yield; 3b: $R f 0.39,12.8 \mathrm{mg}$ yield; 4: $R f 0.50,12.0 \mathrm{mg}$ yield). These were shown to be peoxides by the KI test. ${ }^{18)}$ The spectral data showed that the compounds 2 , 3a, $3 \mathbf{b}$ and $\mathbf{4}$ were stereoisomers of 8a-(1-cyano1,3-dimethyl)butylperoxy- $\delta$-tocopherone. ${ }^{5,7,9)}$ 


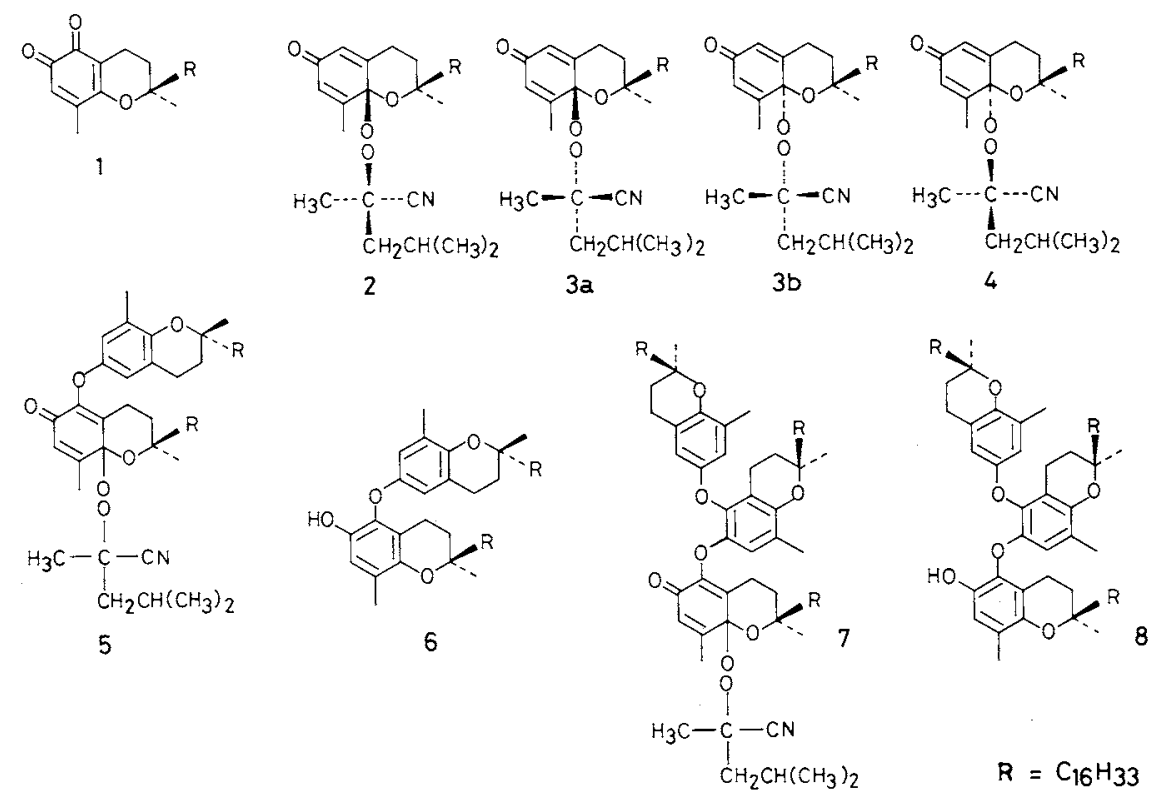

Fig. 2. Structural Formulae of Compounds $1,2,3 \mathbf{3}, \mathbf{3 b}, \mathbf{4}, \mathbf{5}, \mathbf{6}, 7$ and $\mathbf{8}$.

Compound 2, 8a $(S)$-[1(R)-cyano-1,3-dimethyl]butylperoxy- $\delta$-tocopherone. UV $\lambda_{\max }$ (ethanol) $\mathrm{nm} \quad(\varepsilon): 229 \quad(14,300) ;$ IR $v_{\max }$ (film) $\mathrm{cm}^{-1}: 1680,1645 ; \mathrm{MS} m / z: 418\left([\mathrm{M}-125]^{+}\right.$, $3 \%), 402\left([\mathrm{M}-141]^{+}, 95\right), 193(23), 177(53)$, $150(30), 137(100) ;{ }^{1} \mathrm{H}$ NMR $\delta\left(\mathrm{CDCl}_{3}\right)$ : $0.82-0.95(18 \mathrm{H}, \mathrm{m}), 1.08-2.14(26 \mathrm{H}$, broad $\mathrm{m})$, $1.18(3 \mathrm{H}, \mathrm{s}), 1.58(3 \mathrm{H}, \mathrm{s}), 1.85(3 \mathrm{H}, \mathrm{s}), 2.08$ $(3 \mathrm{H}, \mathrm{s}), 2.42(1 \mathrm{H}, \mathrm{m}), 2.90(1 \mathrm{H}, \mathrm{m}), 6.03(1 \mathrm{H}$, s), $6.09(1 \mathrm{H}, \mathrm{s}) ;{ }^{13} \mathrm{C}$ NMR $\delta\left(\mathrm{CDCl}_{3}\right): 16.6$, 19.6, 19.8, 21.5, 22.7 (2C), 23.5, 23.6, 24.5, 24.7 (2C), $24.8(2 \mathrm{C}), 26.9,28.0,32.9$ (2C), 35.5, 37.3, $37.5,37.7$ (2C), 39.4, 41.6, 46.6, 78.0, 78.5, $97.6,120.0,126.3,127.5,153.2,155.4,185.6$; $[\alpha]_{\mathrm{D}}^{25}+23^{\circ} \quad(c=0.19$, ethanol $)$. Compound 3a, $8 \mathrm{a}(S)$-[1(S)-cyano-1,3-dimethyl $]$ butylperoxy- $\delta$-tocopherone. UV $\lambda_{\max }$ (ethanol) nm (c): $229(13,700)$; IR $v_{\max }$ (film) $\mathrm{cm}^{-1}: 1680$, 1645; MS $m / z$ : $418\left([\mathrm{M}-125]^{+}, 3 \%\right), 402$ $\left([\mathrm{M}-141]^{+}, 85\right), 193(23), 177(50), 150(22)$, $137(100) ;{ }^{1} \mathrm{H}$ NMR $\delta\left(\mathrm{CDCl}_{3}\right): 0.840 .93$ $(18 \mathrm{H}, \mathrm{m}), 1.08-2.20(26 \mathrm{H}$, broad $\mathrm{m}), 1.17$ $(3 \mathrm{H}, \mathrm{s}), 1.63(3 \mathrm{H}, \mathrm{s}), 1.99(3 \mathrm{H}, \mathrm{s}), 2.42(1 \mathrm{H}$, m), $3.12(1 \mathrm{H}, \mathrm{m}), 6.01(1 \mathrm{H}, \mathrm{s}), 6.10(1 \mathrm{H}, \mathrm{s})$; ${ }^{13} \mathrm{C} \mathrm{NMR} \delta\left(\mathrm{CDCl}_{3}\right): 16.2,19.7,19.8,21.5$, 22.7 (2C), 23.4, 23.5, 24.6, 24.7, 24.8 (2C), 24.9, $26.9,28.0,32.9,33.0,35.6,37.3,37.5,37.7(2 \mathrm{C})$,
$39.4,41.6,46.7,78.2,78.8,97.4,120.3,126.3$, $127.5,153.9,154.9,185.6 ;[\alpha]_{\mathrm{D}}^{25}-9^{\circ}(c=0.34$, ethanol). Compound $\mathbf{3 b}, 8 \mathrm{a}(R)-[1(S)$-cyano1,3-dimethyl]butylperoxy- $\delta$-tocopherone. UV $\lambda_{\max }$ (ethanol) nm (c): 229 (14,400); IR $v_{\max }$ (film) $\mathrm{cm}^{-1}: 1680,1645$; MS $m / z: 418$ ([M$\left.125]^{+}, 3 \%\right), 402\left([\mathrm{M}-141]^{+}, 83\right), 193(20)$, 177 (47), $150(22), 137(100) ;{ }^{1} \mathrm{H}$ NMR $\delta$ $\left(\mathrm{CDCl}_{3}\right): 0.83-0.95(18 \mathrm{H}, \mathrm{m}), 1.07-1.97(26 \mathrm{H}$, broad $\mathrm{m}), 1.46(3 \mathrm{H}, \mathrm{s}), 1.59(3 \mathrm{H}, \mathrm{s}), 2.07(3 \mathrm{H}$, s), $2.37(1 \mathrm{H}, \mathrm{m}), 2.91(1 \mathrm{H}, \mathrm{m}), 6.03(1 \mathrm{H}, \mathrm{s})$, $6.06(1 \mathrm{H}, \mathrm{s}) ;{ }^{13} \mathrm{C} \mathrm{NMR} \delta\left(\mathrm{CDCl}_{3}\right): 16.8,19.6$, $19.8,20.9,22.6,22.7,23.4,23.5,24.524 .6(2 \mathrm{C})$, $24.8,25.3,25.9,28.0,32.6,32.8,36.3,37.3(2 \mathrm{C})$, $37.4(2 \mathrm{C}), 39.4,43.6,46.6,78.0,78.2,97.7$, $120.1,126.1,127.5,153.3,154.4,185.7 ;[\alpha]_{\mathrm{D}}^{25}$ $-17^{\circ}(c=0.48$, ethanol). Compound $4,8 \mathrm{a}(R)$ [1(R)-cyano-1,3-dimethyl]butylperoxy- $\delta$ tocopherone. UV $\lambda_{\max }$ (ethanol) nm $(\varepsilon): 229$ (14,300); IR $v_{\max }$ (film) $\mathrm{cm}^{-1}: 1680,1645$; MS $m / z: 418\left([\mathrm{M}-125]^{+}, 3 \%\right), 402([\mathrm{M}-$ $\left.141]^{+}, 86\right), 193(35), 177(51), 150(36), 137$ $(100) ;{ }^{1} \mathrm{H}$ NMR $\delta\left(\mathrm{CDCl}_{3}\right): 0.83-0.93(18 \mathrm{H}$, $\mathrm{m}), 1.07-1.84(26 \mathrm{H}$, broad $\mathrm{m}), 1.49(3 \mathrm{H}, \mathrm{s})$, $1.64(3 \mathrm{H}, \mathrm{s}), 1.98(3 \mathrm{H}, \mathrm{s}), 2.39(1 \mathrm{H}, \mathrm{m}), 3.14$ $(1 \mathrm{H}, \mathrm{s}), 6.01(1 \mathrm{H}, \mathrm{s}), 6.09(1 \mathrm{H}, \mathrm{s}) ;{ }^{13} \mathrm{C} \mathrm{NMR}$ $\delta\left(\mathrm{CDCl}_{3}\right): 16.3,19.6,19.8,20.9,22.6,22.7$, 
$23.4,23.5,24.5,24.7,24.8,24.9,25.4(2 \mathrm{C})$, $28.0,32.6,32.8,36.4,37.3(2 \mathrm{C}), 37.4(2 \mathrm{C}), 39.4$, 43.6, 44.7, 78.2, 78.5, 97.5, 120.4, 126.1, 127.6, $154.0,154.8,185.6 ; \quad[\alpha]_{\mathrm{D}}^{25}+22^{\circ} \quad(c=0.45$, ethanol).

Compound 5 was obtained as a yellow oil ( $R f 0.64,75.6 \mathrm{mg}$ yield). This compound was shown to be a peroxide. ${ }^{18}$ From the spectral data, compound 5 was identified to be a mixture of four stereoisomers of 8a-(1-cyano1,3-dimethyl)butylperoxy-5-( $\delta$-tocopheroxy)$\delta$-tocopherone. ${ }^{9)} \mathrm{UV} \lambda_{\max }$ (ethanol) $\mathrm{nm}(\varepsilon): 289.5$ (4680), $234(22,100)$; IR $v_{\max }$ (film) $\mathrm{cm}^{-1}: 1660$; MS $m / z: 818\left([\mathrm{M}-125]^{+}, 4 \%\right), 802(15), 402$ (100), 191 (11), 177 (42), 150 (10), $137(73) ;{ }^{1} \mathrm{H}$ NMR $\delta \quad\left(\mathrm{CDCl}_{3}\right) ; \quad 0.83-0.98 \quad(30 \mathrm{H}, \mathrm{m})$, $1.17-1.95(53 \mathrm{H}$, broad $\mathrm{m}), 1.18(3 / 2 \mathrm{H}, \mathrm{s}), 1.22$ $(3 \mathrm{H}, \mathrm{s}), 1.42(3 / 4 \mathrm{H}, \mathrm{s}), 1.44(3 / 4 \mathrm{H}, \mathrm{s}), 1.62$ $(3 / 4 \mathrm{H}, \mathrm{s}), 1.63(3 / 4 \mathrm{H}, \mathrm{s}), 1.64(3 / 2 \mathrm{H}, \mathrm{s}), 2.03$ $(3 / 2 \mathrm{H}, \mathrm{s}), 2.11(3 / 2 \mathrm{H}, \mathrm{s}), 2.10(3 \mathrm{H}, \mathrm{s}), 2.5-2.9$ $(4 \mathrm{H}$, broad $\mathrm{m}), 6.03(1 / 2 \mathrm{H}, \mathrm{s}), 6.05(1 / 2 \mathrm{H}, \mathrm{s})$, $6.42(1 \mathrm{H}, \mathrm{s}), 6.52(1 \mathrm{H}, \mathrm{s}) ;{ }^{13} \mathrm{C} \mathrm{NMR} \delta\left(\mathrm{CDCl}_{3}\right)$ : 16.2-33.8 (44C), $46.4(1 / 2 \mathrm{C}), 46.6(1 / 2 \mathrm{C}), 75.7$, $78.0(1 / 2 \mathrm{C}), 78.1(1 / 4 \mathrm{C}), 78.2(1 / 4 \mathrm{C}), 78.3$ (1/4C), $78.5(1 / 2 \mathrm{C}), 78.7(1 / 4 \mathrm{C}), 99.3(1 / 2 \mathrm{C})$, $99.4(1 / 2 \mathrm{C}), 112.5,115.2(1 / 2 \mathrm{C}), 115.3(1 / 2 \mathrm{C})$, $119.8(1 / 2 \mathrm{C}), 120.2(1 / 4 \mathrm{C}), 120.3(1 / 4 \mathrm{C}), 121.6$, $126.6,127.2,138.0(1 / 4 \mathrm{C}), 138.2(1 / 4 \mathrm{C}), 138.3$ $(1 / 4 \mathrm{C}), 138.6(1 / 4 \mathrm{C}), 144.1(1 / 2 \mathrm{C}), 144.5(1 / 2 \mathrm{C})$, $147.2,149.5(1 / 2 \mathrm{C}), 149.6(1 / 2 \mathrm{C}), 154.8(1 / 2 \mathrm{C})$, $155.3(1 / 2 \mathrm{C}), 180.3$.

Compound $\mathbf{6}$ was obtained as a colorless oil (Rf $0.70,77.2 \mathrm{mg}$ yield). The structure was identified to be $\delta$-TED by comparing its spectral data with an authentic sample. ${ }^{19)} \mathrm{UV}$ $\lambda_{\max }$ (ethanol) nm $(\varepsilon): 295.5$ (8400); IR $v_{\max }$ (film) $\mathrm{cm}^{-1}$ : 3550; MS m/z: $802\left(\mathrm{M}^{+}, 100 \%\right)$; ${ }^{1} \mathrm{H}$ NMR $\delta\left(\mathrm{CDCl}_{3}\right)$ : $0.83-0.88(24 \mathrm{H}, \mathrm{m})$, $1.08-1.80(46 \mathrm{H}$, broad $\mathrm{m}), 1.21(3 \mathrm{H}, \mathrm{s}), 1.23$ $(3 \mathrm{H}, \mathrm{s}), 2.11(3 \mathrm{H}, \mathrm{s}), 2.15(3 \mathrm{H}, \mathrm{s}), 2.48(2 \mathrm{H}, \mathrm{t}$, $J=7.0 \mathrm{~Hz}), 2.64(2 \mathrm{H}, \mathrm{t}, J=7.0 \mathrm{~Hz}), 4.86(1 \mathrm{H}$, s), $6.36(1 \mathrm{H}, \mathrm{s}), 6.54(1 \mathrm{H}, \mathrm{s}), 6.69(1 \mathrm{H}, \mathrm{s}) ;{ }^{13} \mathrm{C}$ NMR $\delta\left(\mathrm{CDCl}_{3}\right): 11.640 .2$ (40C), 75.3, 75.8, $111.9,115.1,115.3(2 \mathrm{C}), 121.4,123.4,127.7$, 137.1, 141.2, 145.7, 147.2, 149.7.

Compound 7 was obtained as a yellow oil (Rf $0.82,22.5 \mathrm{mg}$ yield). The compound was peroxide. ${ }^{18)}$ The spectral data indicated that compound 7 was a mixture of four stereoisomers of 8a-(1-cyano-1,3-dimethyl)butylperoxy-5$[(5-\delta$-tocopheroxy $)-\delta$-tocopheroxy $]-\delta$ tocopherone. UV $\lambda_{\max }$ (ethanol) nm $(\varepsilon): 291$ (8850), 231 (shoulder); IR $v_{\max }$ (film) $\mathrm{cm}^{-1}$ : $1670 ;$ MS $m / z: 802\left([\mathrm{M}-541]^{+}, 100 \%\right), 402$ (54), 271 (15), 177 (29), 137 (48); ${ }^{1} \mathrm{H}$ NMR $\delta$ $\left(\mathrm{CDCl}_{3}\right): 0.83-0.96(42 \mathrm{H}, \mathrm{m}), 1.07-1.95(72 \mathrm{H}$, broad $\mathrm{m}), 1.07(3 / 2 \mathrm{H}, \mathrm{s}), 1.19(3 / 2 \mathrm{H}, \mathrm{s}), 1.20$ $(3 / 2 \mathrm{H}, \mathrm{s}), 1.21(9 / 4 \mathrm{H}, \mathrm{s}), 1.22(3 / 4 \mathrm{H}, \mathrm{s}), 1.39$ $(3 / 4 \mathrm{H}, \mathrm{s}), 1.40(3 / 4 \mathrm{H}, \mathrm{s}), 1.59(3 / 4 \mathrm{H}, \mathrm{s}), 1.61$ $(9 / 4 \mathrm{H}, \mathrm{s}), 1.99(3 / 2 \mathrm{H}, \mathrm{s}), 2.07(3 / 2 \mathrm{H}, \mathrm{s}), 2.08$ $(9 / 4 \mathrm{H}, \mathrm{s}), 2.09(3 / 4 \mathrm{H}, \mathrm{s}), 2.10(9 / 4 \mathrm{H}, \mathrm{s}), 2.11$ $(3 / 4 \mathrm{H}, \mathrm{s}), 2.30-2.75(6 \mathrm{H}, \mathrm{m}), 5.97(1 / 2 \mathrm{H}, \mathrm{s}), 5.99$ $(1 / 2 \mathrm{H}, \mathrm{s}), 6.38(1 / 4 \mathrm{H}, \mathrm{s}), 6.39(1 / 2 \mathrm{H}, \mathrm{s}), 6.40$ $(1 / 4 \mathrm{H}, \mathrm{s}), 6.47(1 / 2 \mathrm{H}, \mathrm{s}), 6.49(1 / 4 \mathrm{H}, \mathrm{s}), 6.50$ $(1 / 4 \mathrm{H}, \mathrm{s}), 6.63(3 / 4 \mathrm{H}, \mathrm{s}), 6.64(1 / 4 \mathrm{H}, \mathrm{s})$.

Compound 8 was obtained as a yellow oil ( $R f 0.85,22.8 \mathrm{mg}$ yield). The structure was identified to be $\delta$-tocopherol trimer, $5-[5-(\delta$ tocopheroxy)- $\delta$-tocopheroxy]- $\delta$-tocopherol from the spectral data. UV $\lambda_{\max }$ (ethanol) nm (c): 294.5 (11,500); IR $v_{\max }$ (film) $\mathrm{cm}^{-1}$ : 3500; MS $m / z: 802\left([\mathrm{M}-400]^{+}, 41 \%\right), 402(60), 271$ (55), 177 (44), $153(45), 137(100) ;{ }^{1} \mathrm{H}$ NMR $\delta$ $\left(\mathrm{CDCl}_{3}\right): 0.83-0.88(36 \mathrm{H}, \mathrm{m}), 1.07-1.80(69 \mathrm{H}$, broad $\mathrm{m}), 1.20(3 \mathrm{H}, \mathrm{s}), 1.23(3 \mathrm{H}, \mathrm{s}), 1.24(3 \mathrm{H}$, s), $2.04(3 \mathrm{H}, \mathrm{s}), 2.11(6 \mathrm{H}, \mathrm{s}), 2.44(2 \mathrm{H}, \mathrm{m}), 2.65$ $(4 \mathrm{H}, \mathrm{m}), 4.94(1 \mathrm{H}, \mathrm{s}), 6.24(1 \mathrm{H}, \mathrm{s}), 6.41$ and $6.42(1 \mathrm{H}, \mathrm{s}), 6.59$ and $6.60(1 \mathrm{H}, \mathrm{s}), 6.61(1 \mathrm{H}$, s); ${ }^{13} \mathrm{C} \mathrm{NMR} \delta\left(\mathrm{CDCl}_{3}\right): 15.8-40.2(60 \mathrm{C}) \cdot 75.3$, $75.7,75.8,111.4,113.4,114.9,115.4,115.6$, $116.8,121.4,122.7,123.5,127.7,136.7,138.8$, $141.0,142.0,145.4,146.9,147.1,150.8$.

Two different concentrations of $\delta$-tocopherol were reacted with constant AMVN at $37^{\circ} \mathrm{C}$, and the amounts of $\delta$-tocopherol and its reaction products were measured by reversephase HPLC (Fig. 3). At a low concentration of $\delta$-tocopherol, $\delta$-TED (6) formed initially, and then $\delta$-tocored (1), 8a-alkylperoxy- $\delta$ tocopherone (2-4) and 8a-alkylperoxy- $\delta$-TED (5) accumulated with the decrease of $\delta$ tocopherol. In addition to these compounds, trimer (8) and 8a-alkylperoxy trimer (7) were formed at a high concentration of $\delta$ tocopherol. When $\delta$-TED $(1.22 \mathrm{~mm})$ was reacted with $100 \mathrm{~mm}$ AMVN in benzene, $\delta$ - 

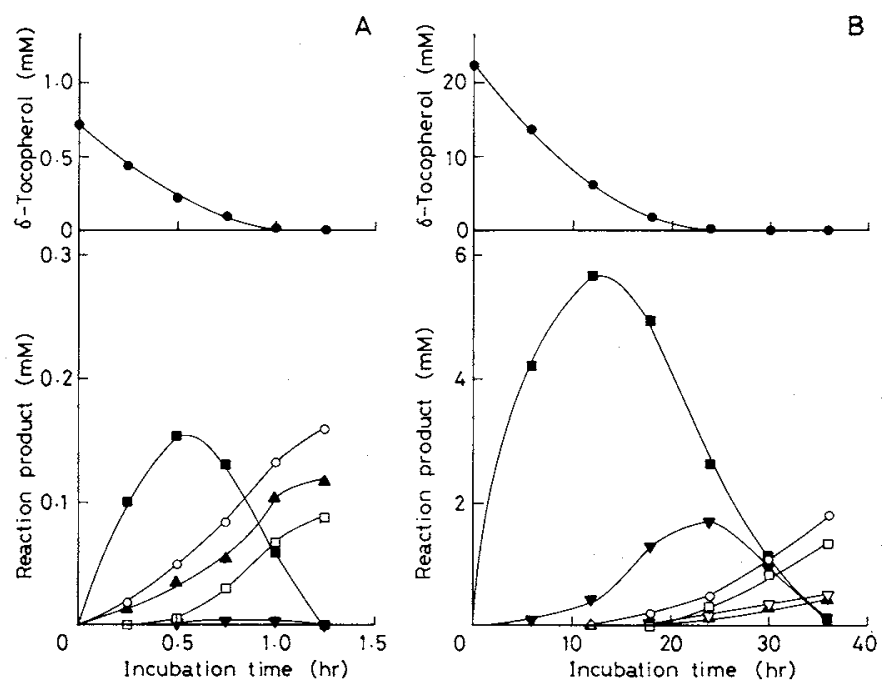

Fig. 3. Course of the Reaction of $\delta$-Tocopherol with AMVN.

A reaction mixture containing $0.73 \mathrm{mM}$ (A) or $22.5 \mathrm{mM}$ (B) $\delta$-tocopherol and $100 \mathrm{mM}$ AMVN in benzene was incubated at $37^{\circ} \mathrm{C}$ - - $-\delta$-tocopherol; - $\bigcirc-, \delta$-tocored; - $\mathbf{-}$-, 8a-alkylperoxy- $\delta$-tocopherone; $\delta$-TED; - $\square-$, 8a-alkylperoxy- $\delta$-TED; $-\boldsymbol{\nabla}-$, trimer; $-\nabla-$, , 8a-alkylperoxy trimer.

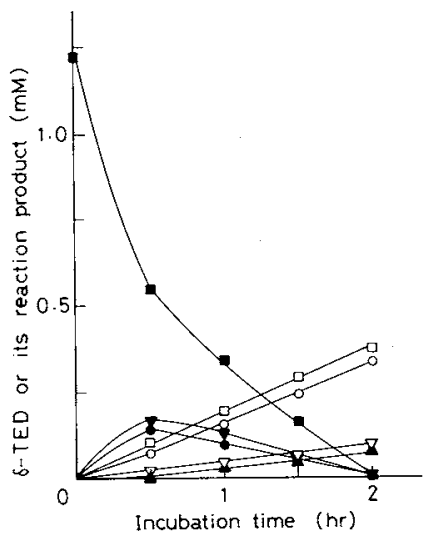

Fig. 4. Course of the Reaction of $\delta$-TED with AMVN. A reaction mixture containing $1.22 \mathrm{~mm} \delta$-TED and 100 $\mathrm{MM}$ AMVN in benzene was incubated at $37^{\circ} \mathrm{C}$. $\delta$-TED; -, $\delta$-tocopherol, $-\mathrm{O}-, \delta$-tocored; $-\mathbf{\Delta -}$, 8a-alkylperoxy- $\delta$-tocopherone; - $\square-$, 8a-alkylperoxy- $\delta$ TED; - - - trimer; -,-- 8a-alkylperoxy trimer.

tocopherol, $\delta$-tocored, 8a-alkylperoxy- $\delta$-tocopherone, 8a-alkylperoxy- $\delta$-TED, trimer and 8a-alkylperoxy trimer were detected (Fig. 4). Although the $\delta$-tocored and 8a-alkylperoxy- $\delta$ TED accumulated with the decrease of $\delta$-TED, the $\delta$-tocopherol and trimer further reacted with another alkylperoxyl radical to produce 8a-alkylperoxy- $\delta$-tocopherone and 8a-alkyl- peroxy trimer, respectively.

\section{Discussion}

The reaction of tocopherols with an alkylperoxyl radical is thought to be a model for the reaction of tocopherols with lipidperoxyl radicals, which occurs in chainbreaking during autoxidation of edible oils. We have already characterized the reaction products of $\alpha$-and $\gamma$-tocopherols with an alkylperoxyl-radical generated from AMVN. ${ }^{5,7,9)}$ They are the covalent adducts of the tocopheroxyl radical with the alkylperoxyl radical and some other products. The reaction products of $\delta$-tocopherol with AMVN were similar to those of $\alpha$ - and $\gamma$-tocopherols; there were $\delta$-tocored (1), 8a-alkylperoxy- $\delta$-tocopherone (2 4), 8a-alkylperoxy- $\delta$-TED (5), $\delta$-TED (6), 8a-alkylperoxy $\delta$-tocopherol trimer (7), and $\delta$-tocopherol trimer (8) (Fig. 2). The 8a-alkylperoxy compounds were produced by the reactions of alkylperoxyl radicals with $\delta$-tocopherol and its reducing dimer and trimer, respectively. $\delta$-Tocopherol biphenyl dimer $(\delta$-TBD $)$ in addition to $\delta$-TED has been reported to be formed during autoxidations of 


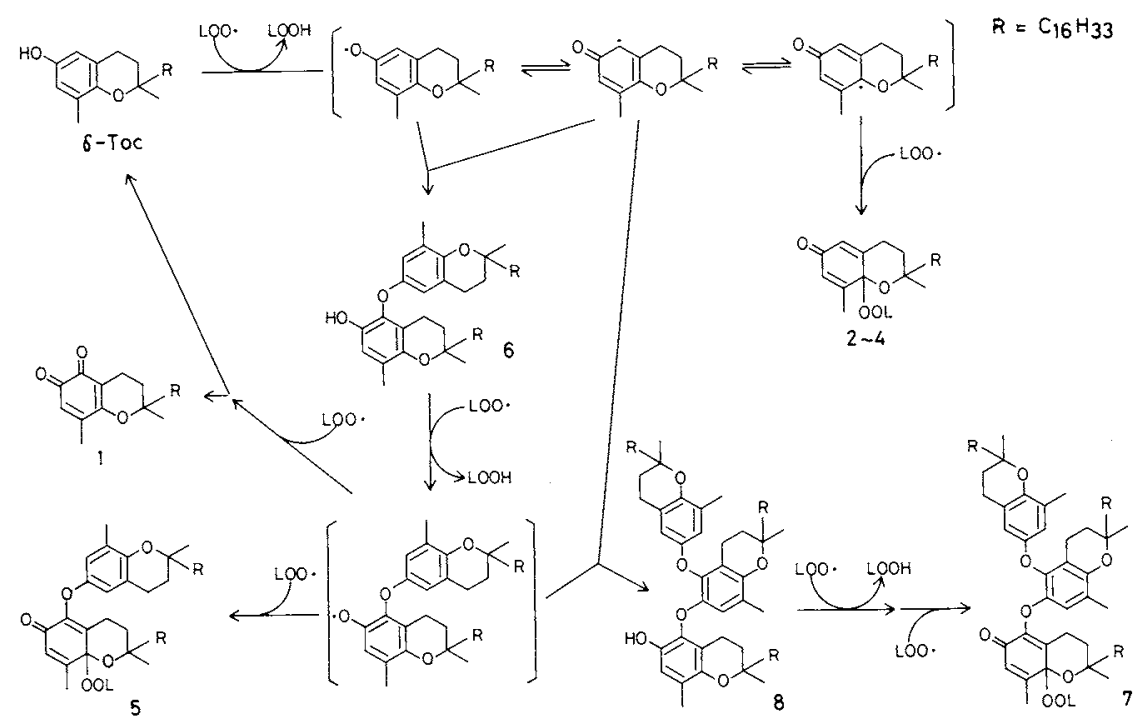

Scheme 1. Pathway for the Reaction of $\delta$-Tocopherol with Peroxyl Radicals.

methyl linoleate ${ }^{12)}$ and triglycerides. ${ }^{13)}$ However, there was no $\delta$-TBD in the reaction mixture of $\delta$-tocopherol with AMVN.

Scheme 1 shows a possible reaction pathway for the reaction of $\delta$-tocopherol with peroxyl radicals. This reaction is started by hydrogen abstraction of the peroxyl radical (LOO -) from $\delta$-tocopherol. The resulting tocopheroxyl radical reacts irreversibly with a second peroxyl radical or with each other to form a covalent adduct or a dimeric compound, respectively. The bimolecular self-reaction of $\delta$-tocopherox$y l$ radical in the presence of oxygen has been reported to be $2 \times 10^{5} \mathrm{M}^{-1} \mathrm{sec}^{-1}{ }^{20}$ ) Therefore, the $\delta$-tocopheroxyl radicals produced can rapidly react with each other to form a dimer. Our results indicated that the $\delta$-tocopheroxyl radicals could react with each other to form the dimer (6) and that the formation ratio of covalent adducts $(2-4)$ was low. Further, the resulting dimer, $\delta$-TED, transferred hydrogen atoms to peroxyl radicals to form $\delta$-TED radicals which, in turn, reacted with another peroxyl radical or with $\delta$-tocopheroxyl radical to form 8 a-alkylperoxy- $\delta$-TED (5) and both $\delta$-tocored (1) and $\delta$-tocopherol or a trimer (8), respectively (Fig. 4). Then the trimer could react with two peroxyl radicals to give 8a-alkylperoxy trimer (7). From these results, not only $\delta$-tocopherol but also its reducing dimer and trimer may scavenge peroxyl radicals during the autoxidation of edible oils.

\section{References}

1) A. L. Tappel, Ann. N. Y. Acd. Sci, 203, 12 (1972).

2) G. W. Burton and K. U. Ingold, Acc. Chem. Res., 19, 194 (1986).

3) J. Winterle, D. Dulin and T. Mill, J. Org. Chem., 49, 491 (1984).

4) S. Matsumoto, M. Matsuo, Y. Iitaka and E. Niki, J. Chem. Soc., Chem. Commun., 1986, 1076.

5) R. Yamauchi, T. Matsui, Y. Satake, K. Kato and Y. Ueno, Lipids, 24, 204 (1989).

6) M. Matsuo, S. Matsumoto, Y. Iitaka and E. Niki, J. Am. Chem. Soc., 111, 7179 (1989).

7) R. Yamauchi, T. Matsui, K. Kato and Y. Ueno, Agric. Biol. Chem., 53, 3257 (1989).

8) R. Yamauchi, T. Matsui, K. Kato and Y. Ueno, Lipids, 25, 152 (1990).

9) R. Yamauchi, T. Matsui, K. Kato and Y. Ueno, Agric. Biol. Chem., 54, 2703 (1990).

10) H. T. Slover, Lipids, 6, 291 (1971).

11) E. Yuki and Y. Ishikawa, J. Am. Oil Chem. Soc., 53, 673 (1976).

12) Y. Ishikawa, E. Yuki, H. Kato and M. Fujimaki, Agric. Biol. Chem., 42, 703 (1978).

13) T. Fujitani and H. Ando, Yukagaku, 28, 896 (1979).

14) R. Yamauchi and S. Matsushita, Agric. Biol. Chem., 41, 1425 (1977).

15) M. Komoda, N. Onuki and I. Harada, Agric. Biol. Chem., 31, 461 (1967). 
16) J. L. G. Nilsson, G. D. Daves, Jr., and K. Folkers, Acta Chem. Scand., 22, 207 (1968).

17) J. L. G. Nilsson, H. Sievertsson and H. Selander, Acta Chem. Scand., 22, 3160 (1968).

18) K. Oette, J. Lipid Res., 6, 449 (1965).
19) Y. Ishikawa and E. Yuki, Agric. Biol. Chem., 39, 851 (1975).

20) G. W. Burton, T. Doba, E. J. Gabe, O. Hughes, F. L. Lee, L. Prasad and K. U. Ingold, J. Am. Chem. Soc., 107, 7053 (1985). 